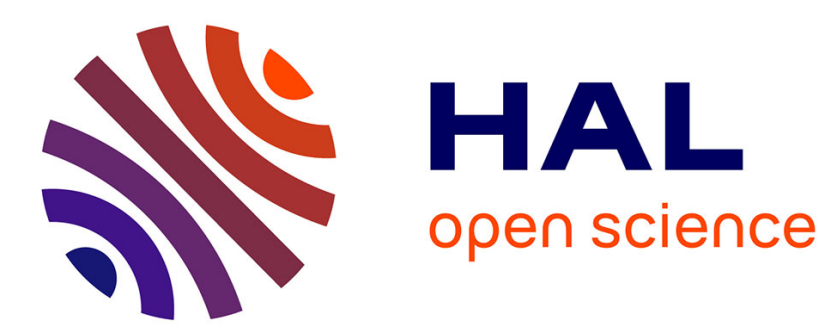

\title{
Coefficient de diffusion dans une chaîne linéaire de Heisenberg à haute température
}

\author{
F. Ferrieu
}

\section{To cite this version:}

F. Ferrieu. Coefficient de diffusion dans une chaîne linéaire de Heisenberg à haute température. Journal de Physique Lettres, 1977, 38 (19), pp.381-383. 10.1051/jphyslet:019770038019038100 . jpa00231401

\section{HAL Id: jpa-00231401 https://hal.science/jpa-00231401}

Submitted on 1 Jan 1977

HAL is a multi-disciplinary open access archive for the deposit and dissemination of scientific research documents, whether they are published or not. The documents may come from teaching and research institutions in France or abroad, or from public or private research centers.
L'archive ouverte pluridisciplinaire HAL, est destinée au dépôt et à la diffusion de documents scientifiques de niveau recherche, publiés ou non, émanant des établissements d'enseignement et de recherche français ou étrangers, des laboratoires publics ou privés. 


\title{
LE JOURNAL DE PHYSIQUE-LETTRES
}

\author{
Classification \\ Physics Abstracts
}

$75.10 \mathrm{~J}$

\section{COEFFICIENT DE DIFFUSION \\ DANS UNE CHAÎNE LINÉAIRE DE HEISENBERG A HAUTE TEMPÉRATURE}

\section{F. FERRIEU}

Institut de Recherche Fondamentale, Département de Recherche Fondamentale, Section de Résonance Magnétique, Centre d'Etudes Nucléaires de Grenoble, 85 X, 38041 Grenoble Cedex, France

(Reçu le 18 juillet 1977, révisé le 29 aô̂t 1977, accepté le 31 août 1977)

\begin{abstract}
Résumé. - Pour une chaîne linéaire de Heisenberg la connaissance des moments jusqu'à l'ordre $2 n=16$ pour des spins $S=1 / 2$, permet d'améliorer la description des fonctions de corrélation de spin à haute température. Nous avons calculé le coefficient de diffusion $D$. La comparaison avec les résultats obtenus par simulation montre que le rapport
\end{abstract}

$$
D / \frac{J}{\hbar} \sqrt{\frac{16 S(S+1)}{3}}
$$

dépend peu de la valeur du spin.

Abstract. - For a linear Heisenberg chain, the knowledge of the 16th order moment for spin 1/2, allows a better description of the time-dependent spin pair correlation function at infinite temperature. The diffusion coefficient $D$ is calculated and compared to the value obtained by a simulation technique $(S=\infty)$. The ratio

$$
D / \frac{J}{\hbar} \sqrt{\frac{16 S(S+1)}{3}}
$$

depends little on the spin value.

Dans les systèmes de Heisenberg unidimensionnels décrits par l'Hamiltonien :

$$
\mathscr{H}=-2 J \sum_{i} \mathbf{S}_{i} \cdot \mathbf{S}_{i+1}
$$

où $J$ est l'intégrale d'échange, le calcul numérique des fonctions de corrélation électronique à deux opérateurs de spin :

$$
F\left(l ; \tau=\frac{J t}{\hbar}\right)=\left\langle S_{i}^{+}(\tau) S_{i+l}^{-}\right\rangle /\left\langle\left|S_{i}^{+}\right|^{2}\right\rangle
$$

a déjà fait l'objet de nombreux travaux [1]. Différentes méthodes de calcul ont été proposées. Certaines se basent fondamentalement sur la détermination des premiers moments $M_{2 n}(q)$ qui interviennent dans le développement de la fonction $F(q, \tau)$, transformée de Fourier dans l'espace des vecteurs d'onde $\mathbf{q}$, de $F(l, \tau)$ :

$$
\begin{aligned}
& F(q, \tau)=\frac{1}{N} \sum_{l} F(l, \tau) \mathrm{e}^{-i q \cdot l}= \\
&=\sum_{n=0}^{\infty}(-1)^{n} M_{2 n}(q) \frac{\tau^{2 n}}{2 n !} .
\end{aligned}
$$

A température infinie, pour une valeur quelconque du spin $S$, les moments $M_{2 n}(q)$ sont connus jusqu'à l'ordre $2 n=6$ [2], [3]. Dans le cas de spin $S=1 / 2$, utilisant une relation de récurrence, Nagao et Miyagi [4] ont effectué le calcul jusqu'à l'ordre $2 n=16$. Ces moments $M_{2 n}(q)$ sont donnés par l'expression :

$$
M_{2 n}(q)=\sum_{m} M_{2 n}^{m}|2(1-\cos q)|^{m}
$$

où les coefficients $M_{2 n}^{m}$ sont tabulés (voir tableau II, référence [4]). A partir de ce résultat, nous avons calculé les densités spectrales :

$$
F(l ; \omega)=\frac{1}{N} \sum_{q} F(q, \omega) \mathrm{e}^{i q . l}
$$

pour $l=0,1,2$.

La fonction $F(q, \omega)$, transformée de Fourier en fréquence $\omega$ de $F(q, \tau)$, est obtenue à partir du formalisme de calcul développé suivant la théorie de la diffusivité généralisée de Bennett, Martin [5] :

$$
F(q, \omega)=\frac{1}{\pi} \frac{D^{\prime}(q, \omega)}{\left|\omega+D^{\prime \prime}(q, \omega)\right|^{2}+D^{\prime 2}(q, \omega)}
$$


où $D^{\prime}(q, \omega)$ et $D^{\prime \prime}(q, \omega)$ sont reliées par la transformation de Kramers-Krönig.

Les moments $\mu_{2 n}(q)$ de $D^{\prime}(q, \omega)$ se déduisent des $M_{2 n}(q)$ par la relation :

$$
\mu_{2 n}(q)=M_{2 n+2}(q)-\sum_{k=0}^{n-1} \mu_{2 k}(q) \cdot M_{2 n-2 k}(q) ;
$$

utilisant les coefficients $M_{2 n}^{m}$, on obtient un développement analogue à l'éq. (3) pour les $\mu_{2 n}(q)$. La connaissance des moments d'ordre supérieur $M_{2 n}(q)$ de $F(q, \omega)$ permet donc de mieux déterminer la fonction $D^{\prime}(q, \omega)$. La méthode utilisée ici est un développement de Cram-Charlier :

$D^{\prime}(q, \omega)=\sqrt{\frac{\pi}{2} \frac{\mu_{0}^{3}(q)}{\mu_{2}(q)}} \sum_{n=0}^{\infty}(-1)^{n} B_{2 n}(q) H_{2 n}(X) \mathrm{e}^{-X^{2}}$

où

$$
\begin{aligned}
B_{2 n}(q)=\sum_{k=0}^{n}(-1)^{k} \frac{1}{2 k !(n-k) ! 2^{2 n-k}} \times \\
\times\left|\frac{\mu_{2 k}(q)}{\mu_{0}(q)}\right|\left|\frac{\mu_{0}(q)}{\mu_{2}(q)}\right|^{k} .
\end{aligned}
$$

Les $H_{2 n}(X)$ sont les polynômes d'Hermites d'ordre $2 n$ et

$$
X=\frac{\hbar \omega}{J} \sqrt{\frac{\mu_{0}(q)}{2 \mu_{2}(q)}}
$$

On obtient dans ce cas pour $D^{\prime \prime}(q, \omega)$ :

$$
\begin{array}{r}
D^{\prime \prime}(q, \omega)=-\sqrt{\frac{2 \pi \mu_{0}^{3}(q)}{\mu_{2}(q)}} \sum_{n=0}^{\infty}(-1)^{n} B_{2 n}(q) \times \\
\times \frac{\mathrm{d}^{2 n}}{\mathrm{~d} X^{2 n}} \mathrm{e}^{-X^{2}} \int_{0}^{X} \mathrm{e}^{t^{2}} \mathrm{~d} t .
\end{array}
$$

Les fonctions $F(l ; \omega)$ calculées ainsi et pour $l=0,1,2$ sont reportées sur la figure 1 . Nos résultats sont comparés dans le cas de la fonction d'autocorrélation $(l=0)$ à ceux obtenus par Tahir-Kehli et Mac Fadden [3] :

- Dans la zone des fréquences $\omega \gtrsim 2 J / \hbar$, on constate une différence notable. La courbe obtenue est nettement inférieure et décroît plus rapidement. L'écart maximum est d'environ $20 \%$ lorsque $\omega \sim 2,5 \mathrm{~J} / \hbar$.

- Aux basses fréquences $(\omega<0,5 J / \hbar)$ un comportement diffusif en $1 / \sqrt{D \omega}$ est imposé par la limite hydrodynamique [5]. Le coefficient de diffusion $D$ est déterminé par:

$$
D=\lim _{q, \omega \rightarrow 0} \frac{D^{\prime}(q, \omega)}{q^{2}}=D_{\mathrm{G}} \sum_{n=0} A_{2 n}
$$

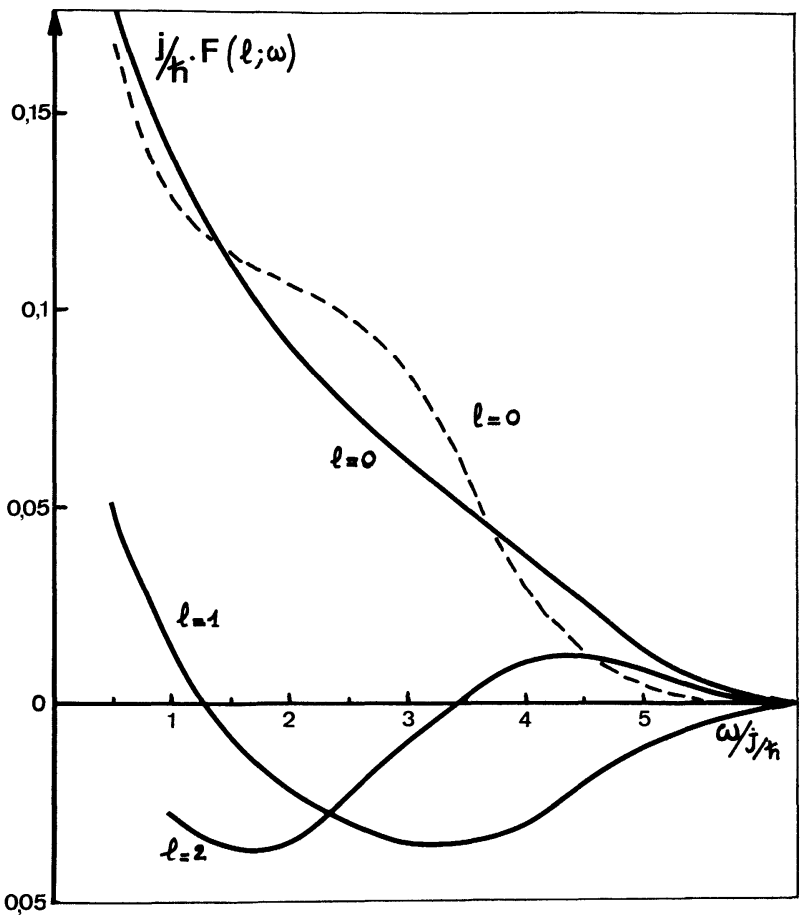

Fig. 1. - Densités spectrales $J / \hbar F(l ; \omega)$ des fonctions de corrélation électronique à deux opérateurs de spin pour une chaîne linéaire de Heisenberg (spin $S=1 / 2$ ) à haute température. La courbe en pointillés représente les résultats du calcul de TahirKehli et Mac Fadden [3].

[Frequency spin-pair correlation functions for a one-dimensional Heisenberg system $(S=1 / 2)$. The dashed curve represents TahirKehli and Mac Fadden's results [3] for $l=0$ (i.e. auto-correlation).]

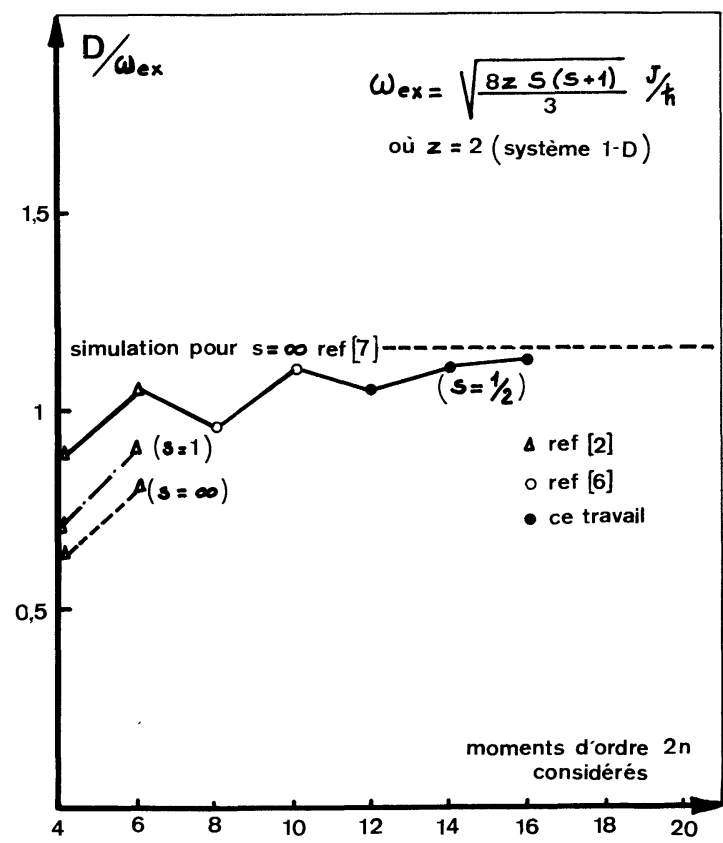

Fig. 2. - Evolution du coefficient de diffusion $D$ suivant les moments $M_{2 n}(q)$ que l'on considère dans le calcul (voir aussi référence [8]).

[Values of the spin diffusion coefficient $D$ according to the moments $M_{2 n}(q)$ used in the calculation (see also ref. [8]).] 
où les coefficients $A_{2 n}$ 'expriment sous la forme :

$$
A_{2 n}=\sum_{k=0}^{n}(-1)^{k} \frac{2 n !}{n !} \frac{M_{2 k+2}^{1}}{2 k !(n-k) !} \frac{1}{2^{2 n+1}}
$$

et $D_{\mathrm{G}}$ correspond à la valeur du coefficient de diffusion dans le cas d'une fonction $D^{\prime}(q, \omega)$ gaussienne [3].

Dans le calcul de Tahir-Kehli et Mac Fadden [3], seul le premier terme $(n=0)$ était considéré. T. Morita [6] a calculé $D$ en tenant compte des cinq premiers coefficients $A_{2 n}$. La connaissance des moments $M_{2 n}(q)$ jusqu'à l'ordre $2 n=16$ calculés par Nagao et Miyagi [4] permet actuellement d'en obtenir huit. C'est là, la limitation de cette méthode. La détermination des moments d'ordre supérieur permettrait d'améliorer la valeur de $D$. Dans la référence [4] des lois extrapolées sont proposées pour certains des coefficients $M_{2 n}^{m}$ [Cf. eq. (3) et (12)]. Aucune ne peut malheureusement s'appliquer à notre cas.

L'évolution de $D$ suivant le nombre des moments peut être appréciée sur la figure 2. La dernière valeur obtenue :

où

$$
D / \omega_{\mathrm{ex}}=1,127
$$

$$
\omega_{\mathrm{ex}}=\sqrt{\frac{16}{3} S(S+1)} \frac{J}{\hbar}
$$

est très proche du résultat obtenu par simulation pour des spins infinis [7] :

$$
D / \omega_{\text {ex }}=1,15 \pm 0,1 .
$$

Ainsi, en ce qui concerne la valeur du coefficient de diffusion $D / \omega_{e x}$, il semble qu'il n'y ait pratiquement pas de différence entre des spins quantiques $(S=1 / 2)$ et des spins classiques $(S=\infty)$.

Remerciements. - L'auteur remercie MM. J.-P. Boucher et M. Nechtschein pour les conseils prodigués ainsi que les fructueuses conversations avec eux tout au cours de ce travail.

\section{Bibliographie}

[1] Voir les références citées dans : Steiner, M., Villain, J., WINDSOR, G., Adv. Phys. 25 (1976) 87-209.

[2] Plaindoux, M., Phys. Lett. 49A (1974) 1. Thèse Grenoble (1975) A.O. 10584 C.N.R.S.

[3] Mac Fadden, D. G., TAHIR-Kehli, R. A., Phys. Rev. B 1 (1970) 3649 et 3671 .
[4] Nagao, N., Miyagi, H., Prog. Theor. Phys. 55 (1976) 21.

[5] Bennett, H. S., Martin, P. C., Phys. Rev. 38 (1965) A 608.

[6] Morita, T., Phys. Rev. B 6 (1972) 3385.

[7] Lurie, N. A., Huber, D. L., Blume, M., Phys. Rev. B 9 (1974) 2171.

[8] Clement, S., Thèse Orsay, 11 mars 1977, p. 33. 